\title{
基于序列操作数据的元素布局预测
}

\author{
李应金，徐鹏飞*，黄惠 \\ (深圳大学可视计算研究中心 深圳 518060) \\ (xupengfei.cg@gmail.com)
}

\begin{abstract}
摘 要: 创建规则的元素布局在许多场景下是常见的任务. 用户在完成该任务的时候往往需要进行大量烦琐的元素 操作. 针对该问题，提出了一种布局预测方法以协助用户高效地创建规则元素布局. 从 SmartDraw 中选取 30 组流程 图模板作为基础数据集并进行数据增强, 以视觉图像和属性编码 2 种形式对数据进行联合编码, 训练以图像字幕网 络为基础框架的神经网络, 使该网络有效地学习到进行元素布局时的规则, 并据此进行布局的预测. 对布局进行预 测时提供包含局部到全局的多个候选结果，保证预测具有可接受的准确率，有效地协助用户完成布局操作. 设计了 包括简单与复杂布局任务的用户调研; 采用量化指标从性能、效率和稳定性对所提方法进行评价和分析. 结果表明, 与传统布局工具相比, 所提方法可显著地减少用户定位元素的次数, 缩减完成布局任务的时间, 有效地提高用户的 布局效率.
\end{abstract}

关键词：布局设计; 布局预测; 机器学习

中图法分类号: TP391.41 DOI: 10.3724/SP.J.1089.2021.18831

\section{Element Layout Prediction with Sequential Operation Data}

\author{
Li Yingjin, Xu Pengfei ${ }^{*}$, and Huang Hui \\ (Visual Computing Research Center, Shenzhen University, Shenzhen 518060)
}

\begin{abstract}
Creating the regular layout of elements is a fundamental task in many scenarios. This task is often tedious since the user has to perform a lot of repeated operations. To address this problem, a layout prediction method is presented to assist the user for the regular layout creation task. 30 groups of flow chart template are collected as the basic data set from SmartDraw and data enhancement operation is applied. These data are encoded in the form of image and property list and are used to train a neural network. The trained network is able to learn the layout patterns and perform the layout prediction. The prediction includes multiple layout candidates in different scales and reaches an acceptable accuracy. Proposed method is tested with a user study including both simple and complex tasks. The evaluation is based on the quantitative analysis of the performance, effectiveness, and stability. The results show that, compared with traditional tools, proposed method is able to notably reduce the number of element positioning operations and the task completion time, and therefore improves the efficiency of layout creation.
\end{abstract}

Key words: layout design; layout predication; machine learning

收稿日期: 2020-12-01; 修回日期: 2021-01-18. 基金项目: 国家自然科学基金(62072316, 61861130365, 61761146002); 广东省自 然科学基金(020A0505100064，2018KZDXM058); 深圳市稳定支持面上项目 (20200812104508001); 深圳市基础研究基金 (JCYJ20180305125709986); 南山领航团队项目(20170003). 李应金(1993-), 男, 硕士研究生, 主要研究方向为计算机图形学; 徐鹏飞 (1986-), 男, 博士, 助理教授, CCF 会员, 论文通讯作者, 主要研究方向为计算机图形学; 黄惠(1977一), 女, 博士, 教授, 博士生导 师, CCF 杰出会员, 主要研究方向为计算机图形学. 
布局是指定物体位置关系的过程. 布局设计 在生活和工作中十分常见. 例如, 使用办公软件对 文章进行排版、制作幻灯片时对内容进行布局; 以 及制作海报、绘制结构图和流程图等，都会涉及布 局设计. 布局是视觉传达的重要手段, 是现代艺术 的重要组成部分. 布局在视觉和心理上对用户有 着重大影响, 其质量将影响用户对内容的评价. 良 好的布局能准确和流畅地传达设计者的意图, 增 加内容的可读性. 布局将各个元素内容之间的关 系转换成空间关系, 它无形之中形成一条脉络, 引 导着用户逐步理解内容. 现有的大多数图形编辑 软件都会提供简单的布局辅助功能帮助用户进行 布局设计, 如最为常见的命令对齐功能.

然而，涉及布局的操作既烦琐又乏味. 在没有 任何布局辅助工具帮助的情况下，想要获得一个 美观的布局几乎无法实现. 现有的布局技术中，基 于命令的布局技术通过重新定位元素的位置, 使 它们在水平或垂直方向上对齐或等距分布; 基于 捕捉的布局技术通过计算其他元素与当前元素的 位置关系，推断它们之间可能存在的对齐和分布 关系, 并在满足条件的情况下微调元素的位置, 使 它满足与其他元素对齐和等距分布的关系; 基于 约束的布局技术通过制定约束重新定位元素的位 置. 各种类别的布局辅助工具都给用户带来便利, 使用户能够快速地完成布局美化任务. 布局技术 的使用，使用户快速上手进行布局设计，高效地完 成布局. 即使是这些布局技术中较为古老的技术, 在很多商业软件中也沿用至今。但是随着社会的 发展，用户对布局技术的要求也逐渐提高，现有的 布局技术已不再满足用户需求. Mackay 等 ${ }^{[1]}$ 认为, 专业用户严重低估了他们在工具的少量操作上花 费的时间，尤其是涉及布局的时间. 这些专业的设 计师认为，他们在设计布局时花费在重新定位图 形上的时间占 $5 \%$ 左右. 但视频记录显示, 他们实 际上花费的时间接近 $30 \%$. Felice 等 ${ }^{[2]}$ 指出, 现有 的布局技术存在缺乏持久性、可预测性和普适性等 问题. 这些报告内容还暴露出一个严重的问题：现 有的布局技术往往只是基于用户的操作对当前的 元素进行修正，这些修正仅仅基于简单的规则，并 且修正程度往往无法预测, 因此用户不得不重新 调整元素的位置.

综上所述，解决在布局设计中存在的问题有 着很重要的学术价值和应用价值. 如果能减少用 户重复定位元素的次数, 将能较大幅度地提升布 局设计的效率. 本文提出一种布局预测方法, 在不
改变现有元素位置的情况下对新元素的位置进行 预测. 本文方法提供多个预测结果, 这些结果可以 作为候选布局为用户提供多个布局样例, 从局部 到全局逐步进行预测，从不同尺度的子序列中获 取预测结果. 本文的布局预测方法存在众多优势: 通过预测新元素的位置引导用户进行布局; 通过 预测的方式直接定位新元素的位置, 显著减少用 户定位的次数; 该方法提供的多个预测结果能直 观比较，便于用户挑选理想布局; 基于神经网络的 布局预测方法可以学习数据中的一般规律; 局部 到全局的预测方式能大大增加方法的鲁棒性, 并 且有效地增加结果的多样性. 本文首次将神经网 络引人布局辅助中, 并创建了用于预测布局的数 据集. 为展示本文方法在实际应用中的效果, 还提 出了一个用于流程图设计的辅助布局系统, 并设 计了 3 个针对性实验评估布局预测方法在布局设 计过程中的辅助效果.

\section{1 相关工作}

布局相关的工作有很多，但与本文类似的布 局工作相对较少. 从提升用户效率和减少用户重 新定位元素的次数的角度分析, 本文方法和其他 布局技术具有一定的相关性. 本文将集中讨论基 于命令的布局技术、基于捕捉的布局技术、基于约 束的布局技术以及一些组合几种技术的组合式布 局技术.

\section{1 基于命令的布局技术}

经典的基于命令的布局技术的起源和前期发 展并无资料记载. 这项技术简单而高效且众多图 形编辑软件都用其辅助用户布局. 2015 年, $\mathrm{Xu}$ 等 ${ }^{[3]}$ 对经典的基于命令的布局技术提出改进, 将其从 一维的对齐与分布关系扩展到二维空间. 2016 年, Felice 等 ${ }^{[2]}$ 的工作将对齐和分布关系看做一类可交 互的对象，改进了传统的对齐与分布工具.

\section{2 基于捕捉的布局技术}

基于捕捉的布局技术具有较长的发展历史. Sutherland ${ }^{[4]}$ 提出的 Sketchpad 系统可以用鼠标自动 捕捉到周围元素, 以协助精确的编辑. Bier 等 ${ }^{[5]}$ 提 出了推断实时对齐和分布对象的算法. 2011 年, Fernquist 等 ${ }^{[6]}$ 的工作将捕捉技术扩展到触屏设备 上. Baudisch 等 ${ }^{[7]}$ 的工作改进了捕捉技术在近距离 放置对象时的表现. 而利用 Felice 等 ${ }^{[2]}$ 提出的工具, 用户能创建一条辅助布局的辅助线, 用来吸附需 进行操作的元素. 


\section{3 基于约束的布局技术}

Sketchpad ${ }^{[4]}$ 是第 1 个可以操作约束的工具. $\mathrm{Juno}^{[8]}{ }^{[D E A L}{ }^{[9]}$ 和 Dunnart ${ }^{[10]}$ 等系统可用于生成满 足用户指定约束的布局. Chimera ${ }^{[11]}$, Pegasus ${ }^{[12]}$, Penguins ${ }^{[13]}$ 等系统注重推理用于布局的约束. DesignScape $^{[14]}$ 可结合用户和系统推断的约束给出布 局建议. $\mathrm{Xu}$ 等 ${ }^{[15]}$ 提出的系统允许用户编辑约束关 系. Wybrow 等 ${ }^{[16]}$ 提出的技术比较了单项约束与多 项约束之间的区别.

\section{4 组合式布局技术}

Gleicher ${ }^{[17]}$ 提出的 Briar 系统组合了捕捉和约 束，将捕捉位置用作计算约束的其中一个参数. Ryall 等 ${ }^{[18]}$ 提出的 GLIDE 系统使用指示器表示约 束对象. Masui ${ }^{[19]}$ 提出的 HyperSnapping 技术将捕 捉位置作为针点表示约束. $\mathrm{Li}$ 等 ${ }^{[20]}$ 和 Lee 等 ${ }^{[21]}$ 提出 基于神经网络的布局生成算法. Zeidler 等 ${ }^{[22]}$ 和 Jiang 等 ${ }^{[23]}$ 提出的系统关注用户界面元素在不同尺 寸屏幕上的布局表现.

\section{2 本文方法}

以神经网络模型作为支撑，本文提出一种基 于序列数据的布局预测方法. 通过直接预测出用 户布置新元素的位置的方式减少用户在布局过程 中重复定位元素的操作次数. 本文方法以用户当 前布置的元素序列为基础收集 2 种形式的数据: (1) 用户在绘制空间中逐一布置元素形成的图像序列 数据; (2) 每个元素的属性参数产生的编码序列. 这 2 种数据将作为输人传递到神经网络模型中得 到多个预测结果. 此外，本文方法采用局部预测与 全局预测相结合的形式，尝试在局部和全局范围 中找到更合理的预测结果.

\section{1 神经网络选择}

本文需要解决从序列数据中预测出新元素位 置的问题, 这需要网络具备从图像数据、序列和多 种数据类型中提取信息的能力. 本文选择经典的 图像字幕网络 ${ }^{[24]}$ 作为网络基本框架, 网络结构如 图 1 所示, VGG-16 $6^{[25]}$ 或 ResNet-5 $5^{[26]}$ 用于从图像序 列中提取较低维表示的特征，这部分特征将会与 属性参数的编码以及上一时刻的输出编码作为注 意力机制的输人. 注意力机制有助于从图像序列 中定位元素的信息，从而可以在这些信息中预测
出新元素的信息. 本文将用 2 种类型的数据输人网 络, 希望网络能学习到这 2 种数据之间的映射表 示. 最后, 本文使用长短期记忆提取序列信息特 征, 再通过全连接层将最后的特征表示解码.

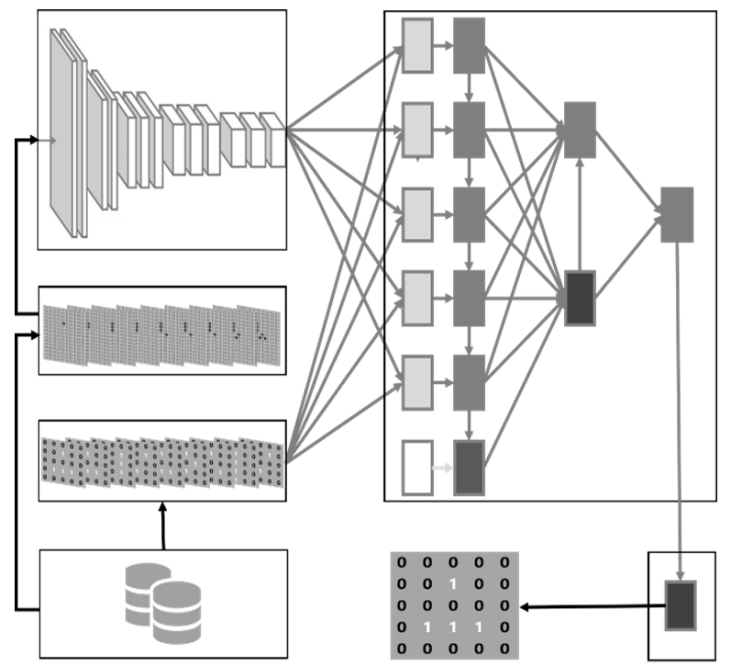

图 1 网络结构图

\section{2 数据集}

最近几年的关于布局辅助技术的工作并没有 提供可以用作神经网络训练的数据集. 本文从相 关网站上收集到数据模板, 并将其制作为本文的 数据集.

\subsection{1 数据获取}

从 SmartDraw ${ }^{\circledR}$ 官网下载了 65 组流程图设计模 板. SmartDraw 是一套由 SmartDraw.com 开发的图 表绘制软件，它能绘制流程图等不同形式的商业 图表，是目前市面上较为流行的图表绘制软件之 一. 为帮助用户快速进行流程图设计, 其内置了多 个流程图的模板. 本文从中选取 30 组模板作为数 据集的基础数据, 个别模板如图 2 所示.

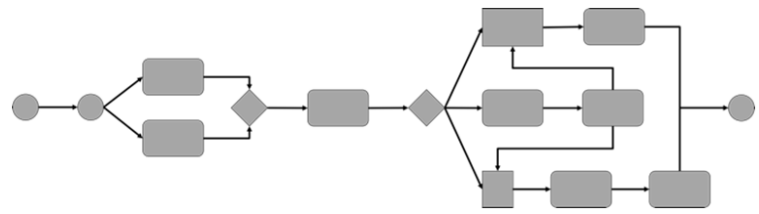

图 2 原始图模板展示图

\section{2 .2 数据增强}

由于从网站得到的模板数据较少, 为使网络 获得较为可靠的预测结果, 需要对每个模板进行 数据增强. 对于某个模板, 数据增强后需保证数据 
仍可被识别为同一个模板; 数据集以改变数据元 素的位置为主进行数据增强. 本文使用网格的形 式对数据位置加以限制, 使每个元素的位置只能 出现在某个格子中. 格子的尺寸可以调节，但其必 须大于元素的尺寸. 网格化后的数据可以对元素 进行固定长度的编码, 元素位置关系变化以网格 作为度量单位. 读取模板文件的信息得到如图 3a 所示的某个模板, 进行如下操作：首先固定元素的 尺寸，使每个元素的包围盒保持一致; 其次在水平 方向和垂直方向上进行分组，得到一个水平方向 和垂直方向的数组, 以此来保持每个模板的主要 结构; 然后将在水平分组和垂直分组中重新计算 每个元素的位置, 使元素间隔关系为 0 (以网格为 单位), 从而得到一个水平和垂直方向上元素间隔 都为 0 个网格的模板. 如图 $3 b$ 所示, 元素依然保持 原模板结构，其余的位置关系变换都以此 0 间距的 模板为基础. 为增加数据的多样性, 本文同时对数 据进行如下操作：随机设置水平或垂直分组的间 隔数据(以网格为单位), 使数据中元素的相对位置 上的变化如图 3c 所示; 随机设置初始偏移, 使每 个元素在图片内的位置随机分布如图 3d 所示. 即 使是在带有强烈顺序信息的布局设计中，也没人 会完全按照流程图的顺序进行布局设计。

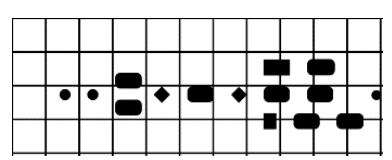

a. 网格化前

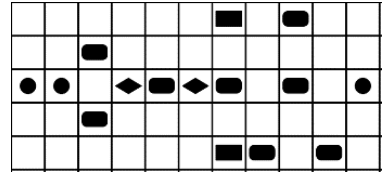

b. 网格化后



c. 间距变化前

d. 间距变化后
图 3 数据增强图

为模拟这种实际的操作情形，本文随机调换 除初始和结束位置的 2 个元素以外的其他相邻元 素，使元素出现的顺序在局部上是随机的(有较小 的概率偏向全局随机). 在得到最终的元素序列后, 将元素序列按照不同的长度分解成子序列，长度 范围在 3 和最短序列的长度之间. 在训练时, 对于 图像数据本文重设图像尺寸为 256 像素, 并且将图 片转成单通道的灰度图; 然后反转黑白颜色且把 像素的取值范围从 $[0,255]$ 变换到 $[-1,1]$. 在生成图 像序列的同时，每个序列元素其所属模板类别、类
型信息、尺寸信息和位置信息也会被记录并进行编 码. 这些信息最终组合成一个编码，用来描述每个 元素的属性

\section{3 多数据联合策略}

本文的数据有 2 种不同的形式, 一种是视觉信 息数据, 另一种是属性编码数据. 用户在评价布局 设计时, 大多以视觉为主. 在不借助外部工具的情 况下，用户仍然能够从视觉角度评价每个布局的 质量，这是一种很自然地感受布局的方式. 为还原 这种视觉评价方式，本文获取用户在进行布局设 计时的视觉信息, 并将其制作成图像序列, 以作为 布局预测的输人之一. 图像序列数据的好处在于 其能够保存各个元素之间的空间关系和时序关系. 传统方法往往使用元素的位置等属性作为其算法 的输人, 本文方法也使用属性参数, 将元素的位置 参数转化成其所在绘制空间中网格的编码, 以此 来表达元素的位置属性. 本文联合这 2 种不同形式 的数据作为输人, 以提高数据自身的表达能力. 图 像通常表示为特征提取器的像素强度或输出, 而 属性编码则表示为离散的向量. 不同信息资源的 统计特性不同，联合不同模态的数据进行训练更 能发现不同模态之间的关系，以便更好地引导模 型进行训练.

\section{4 多结果预测策略}

用户在创建新布局时，对每个元素都会执行 明确的操作, 并且需要手动添加新元素. 用户想要 获得最佳的布局, 往往需要创建多个候选布局, 并 从中挑选出合适的布局. 受此启发, 本文在用户每 次创建元素时提供多个预测结果作为候选, 用户 以这些结果作为指导逐步完成布局. 本文将整个 绘制空间划分成多个网格, 将求解元素的位置转 换成如图 4a 所示的求解其所在网格的位置, 网格 的数目是有限的且其位置固定, 本文对每个网格 中是否存在元素进行标记. 如果存在, 标记为 1 ; 否则标记为 0 . 整张画布的元素分布情况使用固定 长度的编码就能表示, 如图 $4 \mathrm{~b}$ 所示.

同样地，每个元素的位置描述也能使用相同 长度的编码表示. 这种表示还存在另外一个意义， 即该元素在各个网格上的概率大小, 这种方式把 计算每个元素的位置问题逐渐转换成计算每个元 素落在各个网格上的概率问题. 这种表达方式还 有另外一个好处: 在预测新元素位置时, 算法可以 依据概率大小向用户提供多个预测候选值. 多个 预测候结果为用户提供了多个不同的布局样例, 以协助用户完成布局. 


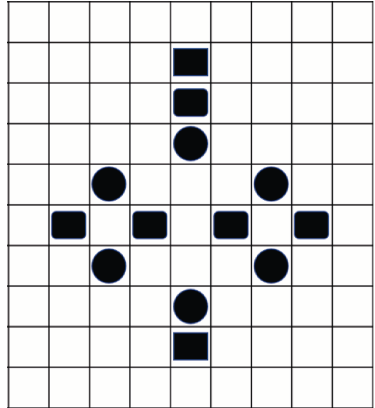

a. 编码前

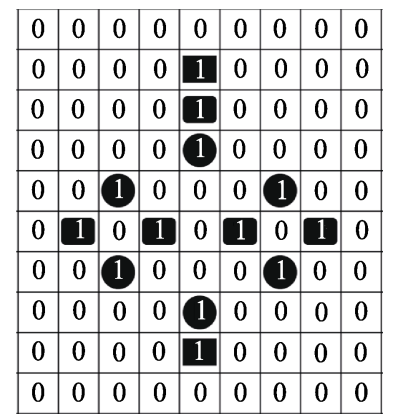

b. 编码后
图 4 编码示意图

\section{5 多尺度预测策略}

如图 5a 所示，当用户按图中顺序布置元素时， 最简单有效的方法是把整个元素序列作为输人然 后预测出最终结果, 如图 5d 中红色部分所示. 但 是在实际的使用过程中, 由于用户最终的布局是 未知的，所以无法确切地知道用户新元素的布局 位置. 另外, 用户在布置新元素时, 所参照的元素 也无从得知, 不同数目及不同顺序的元素排列都 可能预测出不同的结果. 如图 $5 \mathrm{~b}$ 和图 $5 \mathrm{c}$ 所示, 使 用序列 $\{7,8,9,10\}$ 和 $\{4,5,6,7,8,9,10\}$ 进行预测, 得出的预测结果不同, 如图 5 中的红色部分. 可以 看到，不同长度的序列所表达的布局关系是不一 致的. 而在实际的数据中, 遇到的局部关系和全局 关系会更加复杂多样.

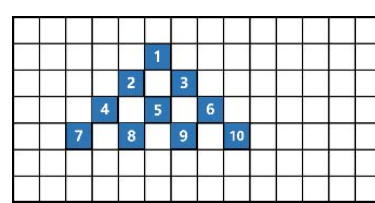

a. 全部序列

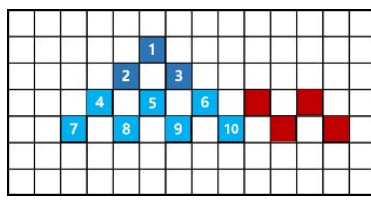

c. 局部序列(4 10)参与预测

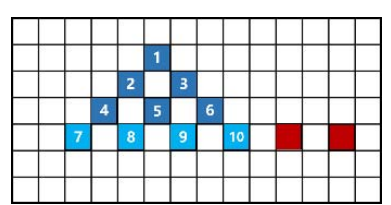

b. 局部序列(7 10)参与预测

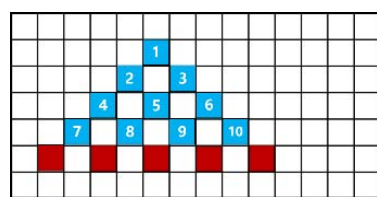

d. 全部序列参与预测
图 5 局部到全局预测图

本文采用全局与局部相结合的方法提高预测 的命中率. 给定输人序列, 布局预测方法会从此序 列中抽取不同长度的子序列进行预测，尝试寻找 从局部到全局的预测结果. 不同长度的子序列代 表不同程度的局部信息，越接近原有的长度，说明 序列的预测结果越接近全局，而局部范围预测更 能获取局部规律. 当用户在进行自主创作时, 他并 不会依赖于某个模板而是在进行个性化创作，这 会导致基于全局范围的预测性能大大下降. 多尺
度预测能捕捉到用户在不同尺度上的设计习惯， 从而给出更多合理的预测建议.

\section{3 应用系统}

本文基于提出的布局预测方法开发出一个用 于流程图设计的系统. 本文使用创建的训练集对 神经网络进行训练得到网络模型, 系统使用模型 预测用户添加新元素的位置. 系统交互流程图如 图 6 所示, 用户通过交互布置元素, 其中的交互操 作包括命令对齐和捕捉对齐等. 当用户定位元素 操作完成时, 系统生成输人数据, 并通过数据处理 模块将数据转换为模型输人数据的形式, 随后将 其传递到网络模型模块中. 网络模型产生输出数 据, 产生的输出数据仅仅是新元素在各个网格的 概率, 因此需要对数据进行后处理, 将前 $k$ (实验 设定 $k=10$ ) 个概率提取出来作为预测的候选值. 接着可视化模块将各个候选值可视化出来显示在 系统中，用户通过交互选择需要的可视化结果.

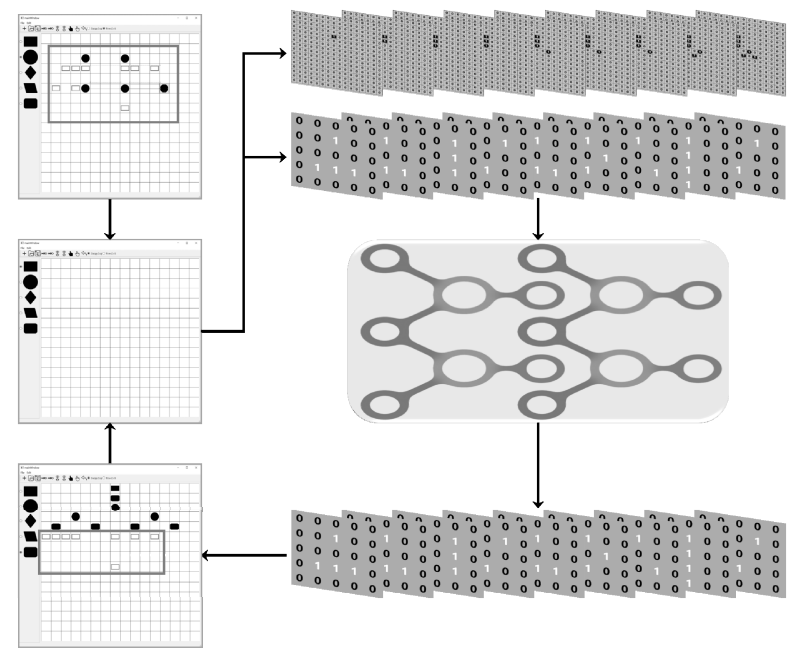

图 6 系统交互流程图

\section{1 应用场景选取}

本文选取流程图设计作为主要使用场景。流 程图中各个元素之间存在着一定次序, 元素之间 的布局关系(如水平、垂直、等间距等)很弱，且布 局关系较为复杂. 因此, 流程图是一个典型的应用 场景. 但是流程图的次序在绘制过程中的作用较 弱，每个人根据绘制习惯的不同，并不会完全按照 流程图的顺序进行绘制.

\section{2 模型训练}

在训练时, 本文使用 $L_{2}$ 损失函数计算输出结 果与目标编码之间的差异, 并进行优化. 训练过程 
中从数据集中随机抽取 10 个模板，每个类别 10264 个序列数据, 其中 8000 个作为训练, 2264 个作为测试. 实验设定序列长度为模板中最长序 列的长度 16. 初始学习率设为 0.0001 , 学习率在 每完成 1 次迭代后衰减, 衰减因子为 0.99 , 直至学 习率降到不大于 0.00001 后保持不变, 训练迭代 200 次.

\section{3 功能介绍}

本文系统设置了几种常用的命令对齐方式: 左对齐、右对齐、顶端对齐、底端对齐分别以最左 边元素、最右边元素、最顶端元素、最底端元素对 齐, 且以上对齐方式均以左上角的坐标为基准进 行对齐; 水平中对齐以选中元素的平均横坐标对 齐, 垂直中对齐以选中元素的平均纵坐标对齐, 以 上对齐方式以选中元素的中心坐标为基准对齐. 左对齐过程如图 7 所示, 用户首先框选需要对齐的 元素，如图 7a 所示，随后单击“对齐”按钮执行左 对齐命令, 所有选中的元素以最左边的元素的左 边界为参照位置, 如图 7b 所示.

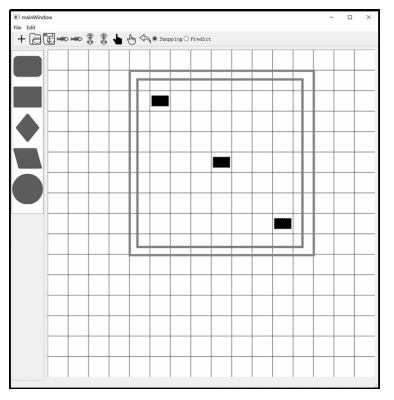

a. 对齐前

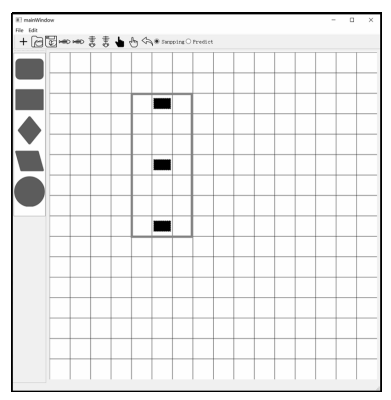

b. 对齐后
图 7 命令对齐功能(左对齐)

捕捉分为对齐捕捉和等距分布捕捉 2 种. 对齐 捕捉计算任意 2 个元素之间的距离关系，距离关系 在一定误差范围内认为元素存在位置对齐关系. 存在对齐关系的 2 个元素之间，系统会绘制一条对 齐的关系线提示用户．在绘制对齐关系线时，系统 将考虑元素的前后关系, 并计算 2 个端点的相对偏 移，以保证绘制线不穿过元素本身. 等距分布捕捉 将计算任意 2 个元素与当前元素之间的间距关系. 等距关系分为垂直关系和水平关系，在确定 3 个元 素之间存在等距关系后，系统也会绘制 2 个带箭头 的虚线提示它们存在等距关系. 若位置对齐和等 距分布同时存在，为避免位置对齐线和等距分布 线重合而影响用户判断，等距分布线将向外偏移 一定距离. 捕捉功能如图 8 所示, 捕捉到对齐或等 距分布关系时，系统都会绘制出对应的提示线. 对 齐捕捉如图 8a 所示, 对齐捕捉功能主要捕捉元素
左上角、右下角以及元素中心的对齐. 等距捕捉如 图 $8 \mathrm{~b}$ 所示, 等距分布线以中间元素为基准进行绘 制, 表示两边的元素以中间元素为纽带形成等距 分布关系。

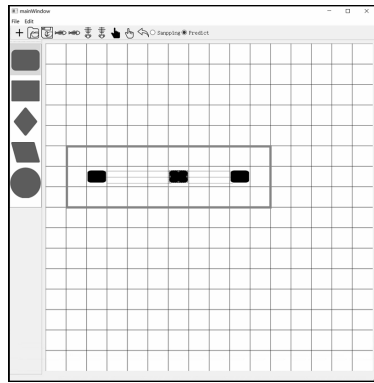

a. 对齐捕捉

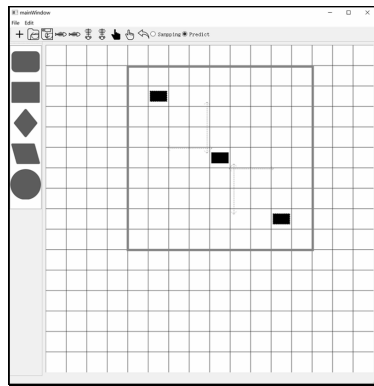

b. 等距捕捉
图 8 捕捉功能

为了将各个元素连接组成完整的流程图, 系 统还提供元素间的连线功能. 每个元素在创建时 附带 4 个连接节点, 每 2 个元素相互连接的节点会 创建一个连接器. 连接器由 5 条短线段组成, 这 5 条线段都可以自由伸展，但只能在水平或垂直方 向上移动. 如果一条线段被移动, 则连接器其他 的线段会伸展连接这条线段. 连接器最多提供 4 个拐点帮助用户完成元素之间的连接, 用户可以 移动线段以避免线段和元素重叠. 如果移动线段 会增加连接器线段的数量或增加的拐点数目超过 4 个, 则这条线段不能移动. 线段连接是动态的, 会随制定的元素移动而移动. 用户单击 “连线” 按 钮, 连接元素过程如图 9 所示, 其中图 9a 表示选 择起始元素, 图中选择了元素底端的连接节点; 图 $9 \mathrm{~b}$ 表示选择结束元素, 图中选择了左端的连接 节点; 图 9c 表示构建连接器并生成线段连接 2 个 元素; 图 9d 表示控制连接器中线段的移动.

当用户输人元素数目超过 3 , 布局预测功能启 用. 系统将输人数据分成不同长度的子序列输人 网格中, 以获得在不同的局部规模中得到的预测 候选项, 系统优先从不同尺度的预测结果中抽取 概率较大的选项, 并从这些候选项中篮选出前 $k$ 个作为最后的建议位置显示出来. 不同长度的子 序列能获得不同尺度的局部预测建议，用户将得 到更加多样的预测候选结果. 在实际应用中, 由于 无法判断用户最终的布局设计, 局部预测变得比 全局预测更加实用, 多尺度的预测结果能将用户 在多个尺度上的布局习惯预测出来作为候选. 网 络模型生成预测候选结果时, 系统获取到前 $k$ 个 预测候选结果并计算这 $k$ 个候选结果所对应的网 
格, 预测候选结果可视化效果如图 10 所示.

由于最终的预测结果来自不同长度的子序 列, 导致候选结果的次序逐渐弱化. 系统使用相 同的颜色可视化这些结果. 同时, 可视化多个预 测结果, 不仅为用户提供了多个布局样例, 而且 还能形成直观的对比效果, 从而引导用户寻找到 最合适的布局方案. 可视化的元素并不真正存在 于布置序列中, 因此不会影响场景中已经布置好 的元素. 预测候选结果可以选择多个或不选择, 如果用户选择多个预测候选结果, 则选择顺序就 代表用户布置元素的顺序; 如果不选择任何元素 或已经完成选择, 则需要在空白处右击取消选择 状态.

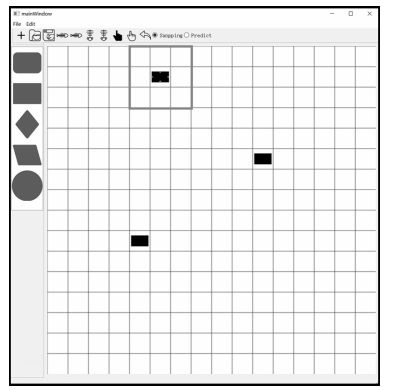

a. 创建连线起点

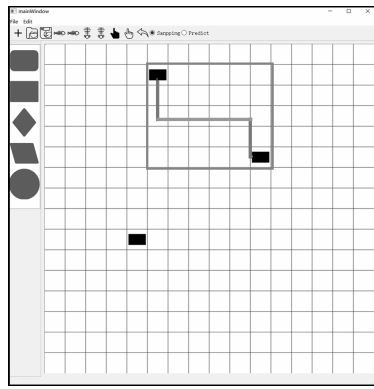

c. 创建连线

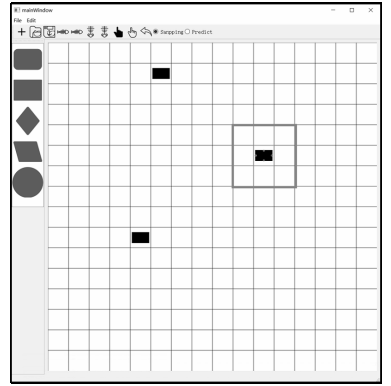

b. 创建连线终点



d. 编辑连线
图 9 连线功能

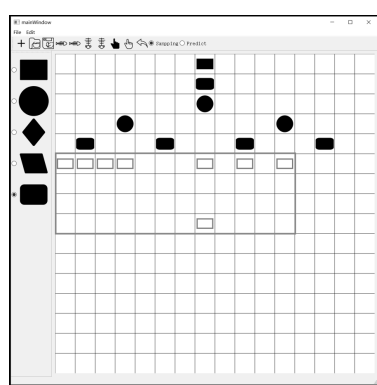

a. 可视化结果 1



b. 可视化结果 2
图 10 可视化结果

\section{4 用户调查与结果分析}

本文采用用户调查的方法评估所提出的布局
预测方法. 本文设计针对性的实验并招募用户进 行测试，每项测试包含不同的评估指标. 在测试过 程中, 系统收集用户各项指标参数作为评估的依 据. 最后通过量化分析各项指标评估本文方法.

\section{1 访 谈}

本文邀请了 15 个人参与用户调查, 他们的年 龄分布在 20 40 岁, 其中大部分人为 20 30 岁. 参 与用户调查的用户中, 有 5 人是专业的平面设计 师, 还有 5 人经常使用其他的图形编辑工具, 另外 5 人很少使用图形编辑工具. 所有人都至少使用过 一种图形编辑工具.

对招募的用户进行一对一访谈, 以便更好地了 解他们在图形编辑工具中遇到的问题. 参与者中有 超过一半的人经常使用高级图形编辑工具, 这些人 中又有一半的人属于专业的图形界面设计师. 访谈 时请他们展示最近的项目, 要求他们回顾特定问 题, 并进一步了解他们如何解决这些问题.

本文使用扎根理论方法 ${ }^{[27]}$ 对收集的访谈内容 进行分类. 扎根理论为分析定性数据提供了一种 系统的方法, 该分析不是检验性假设, 而是由一系 列开放性问题和严格的策略指导数据收集. 该方 法的目标是对出现的现象提供丰富的描述, 先确 定每段访谈中的特定问题, 并与其他访谈内容进 行了交叉核对. 这为本文提供了这些参与者在布 局设计时面临的关键问题的列表, 并指出了这些 问题存在的普遍性.

在访谈过程中, 参与者可以自由选择访谈地 点, 可以是他们自己家里、实验室, 也可以是工作 的地方. 在访谈前会聊一些与采访内容无关的东 西, 甚至会和他们做一些放松的小游戏, 目的是尽 量引导用户快速地放松下来. 同时, 鼓励他们展示 他们的布局设计项目, 在布局情境中参与访谈, 更 能帮助找到系统中的问题. 系统设计人员参与到 他们的布局设计日常中, 了解他们在布局过程中 都在做些什么, 以及他们为什么这样做. 对参与者 进行编号, 其中 $P_{1} \sim P_{5}$ 属于专业的布局设计用户, $P_{6} \sim P_{10}$ 属于熟练掌握 PPT 等办公软件或图形编辑 工具的用户, $P_{10} \sim P_{15}$ 则较少使用图形编辑工具, 布 局设计经验较少. 对所有招募人员进行访谈并总 结其言论, 得到以下结果.

其中一部分人 $\left(P_{1}, P_{4}, P_{6}, P_{8}, P_{10}, P_{11}, P_{13}, P_{14}\right)$ 希望布局设计工具能够协助他们完成布局. 对于 一些不经常参与布局任务的用户，工具能够提供 相应的帮助很重要. $P_{4}$ : “我通常在布局设计时没有 灵感, 我会一开始尝试几个我经常放置的位置, 看 
看是否适用于现在的布局情景.” $P_{8}$ : “我经常会使 用软件提供的一些模板, 但是有时候在设计过程 中, 我不清楚怎样设计会更好, 我希望软件能像秘 书一样给出一定的建议.” $P_{13}$ ：“我有时候觉得布局 设计真的很麻烦, 不停地移动元素, 如果工具能够 提供一种类似于软件的新手教程的提示，让我不 用拖动元素，只需要不停单击“下一步”按钮就能完 成布局, 那真的很不错”.

另一部分人 $\left(P_{3}, P_{5}, P_{7}, P_{8}, P_{9}, P_{10}, P_{14}, P_{15}\right)$ 认 为为了得到更好的布局, 需要创建许多额外的潜 在布局进行对比，且这些布局无法直接对比. $P_{5}$ : “在布局设计时会做很多无用功，因为我有太多想 尝试的方案，但是我不得不逐个去尝试它们. 很多 时候我又得倒回去选择最初的那个方案. 有时候 我会通过创建多个元素摆放在想要去尝试摆放的 位置, 然后从中挑选出更好的方案, 最后再一个个 删掉它们.” $P_{14}$ : “我觉得我有很大一部分时间都是 在移动元素，我通常都是通过不断累积的“经验”布 置元素，通过不断尝试不同的摆放位置并凭借记 忆从中选择较好的布局方案. 我有时候通过撤销 和重做功能来回切换不同布局方案进行对比.”

还有一部分人 $\left(P_{2}, P_{3}, P_{4}, P_{5}, P_{7}, P_{9}, P_{15}\right)$ 认为 他们使用的工具不够 “智能”, 不能够识别他们的 行为习惯. $P_{2}$ : “我经常做一些重复的东西，但是使 用的工具却不能识别到，导致我不得不从头开始 设计. 我使用的工具要是能更智能一点就好了.” $P_{7}$ : “我在进行个性化的布局设计时效率会变得很 低，因为没有适合的布局辅助工具给我使用. 我经 常会复制我之前的一些作品到现在的布局环境中, 然后再逐个调整.” $P_{15}$ ：“有时候我想做一些不太寻 常的设计，但是我使用的工具不能辅助我快速实 现它们，这导致我手动调整元素的时间会更长.”

通过访谈, 本文总结出现有的图形编辑工具 存在的局限. 现有的编辑工具主要有以下问题.

（1）布局过程中缺乏引导和协助.

（2）候选布局无法直接相互比较.

（3）无法捕捉一般性或个性化的布局规律.

\section{2 调 研}

本文设计了 3 个实验评估布局预测方法，以检 验其在辅助用户布局时发挥的作用. 为了比较全 面地评估，本文从性能、效率、稳定性 3 方面对其 进行考虑. 对于每个实验，系统从模板中抽取 10 个模板作为用户调查的数据, 这 10 组数据来自不 同长度的模板, 长度范围为 7 15. 将这 10 组数据 按照序列长度分为简单和复杂 2 类(长度大于 10 为
复杂，小于或等于 10 则为简单).

对于实验 1 和实验 2 , 系统随机从简单和复杂 2 类数据中分别抽取 2 组数据和 3 组数据进行实验. 对于实验 3, 给出 10 个不存在于训练集中的模板, 其中 5 个为简单模板, 5 个为复杂模板. 用户同样 从中抽取 2 组简单数据和 3 组复杂数据. 用户根据 流程图的模板自由设计该流程图的变体, 其中元 素的绘制顺序和位置都由用户自由指定.

本文邀请另外 15 人参与实验，他们的年龄分 布在 20 40 岁, 其中大部分人为 20 30 岁. 参与实 验的用户中， 7 人经常使用布局相关工具，另外 8 人较少使用布局相关的软件. 同样对参与者进行 编号, 其中 $P_{1} \sim P_{7}$ 属于经常使用图形编辑工具的用 户, $P_{8} \sim P_{15}$ 则较少使用这些工具.

在每个参与者开始实验前，系统设计人员都会 对其进行培训，包括界面介绍、功能介绍及演示等， 最后将向参与者展示完整的布局过程, 在确保参与 者已经掌握实验所需的操作后才开始进行实验.

\subsection{1 实验平台}

本文以开发的应用系统为基础进行实验，并 在应用系统中增加了额外的显示区域，用来显示 和提示用户当前需要完成的布局任务. 抽取的序 列数据显示在系统界面的右侧, 用户可以通过鼠 标左右键控制数据序列, 左键表示下一个元素, 右 键表示上一个元素. 如图 11 所示, 右侧部分为显 示区，左侧部分为用户布置元素的操作区. 用户根 据显示区的提示在操作区进行操作, 在相应位置 布置元素，如果预测的位置和显示区的位置一致 则表示预测命中. 用户在预测位置选择元素或布 置元素，然后单击显示区进行下一步操作.

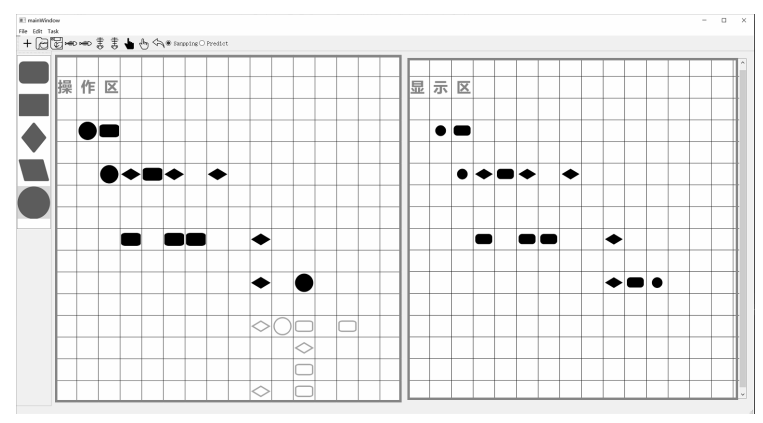

图 11 实验平台展示图

\subsection{2 方法性能分析}

首先验证布局预测的布局方式是否能有效地 帮助用户. 布局预测方法的主要功能是预测用户 新元素的摆放位置. 如果能预测正确, 用户就能直 接选择该位置布置新元素，而避免拖动、对齐等麻 
烦的操作. 用户完成布局的过程中预测方法成功 预测所占的比重将被统计. 这项实验将评估本文 方法在布局设计中发挥的辅助作用.

系统将抽取的 5 组数据分别展示出来，用户根 据数据序列所示的顺序绘制元素. 绘制过程中系 统会不断给用户提供多个预测建议, 用户从中选 择一个或一个以上作为新元素定位的位置. 用户 可以采纳或忽略这些建议.

对布局预测方法最直接和有效的评估依据就 是观察用户在布局设计过程中是否采纳了系统的 预测建议，以及采纳的次数在整个布局过程中所 占的比重大小. 系统将记录预测命中次数这项重 要指标, 即用户选择系统所提供的建议的次数. 这 项指标直接确定本文方法在整个设计过程中所发 挥的作用.

实验结果如图 12 所示，可以看出，在本次实 验中简单组最高平均命中次数为 5.5 次, 最低平均 命中次数为 3.5 次; 复杂组最高为 9.34 次, 最低为 6.67 次. 简单组预测命中率大于 0.50 的仅有 3 组, 分别为 $P_{2}, P_{11}, P_{13}$. 而复杂组仅有一组 $\left(P_{6}\right)$ 未达到 0.50 , 说明简单组的命中率相对低于复杂组命中 率. 详细的命中率数据如图 13 所示. 图中红色折 线代表的复杂组命中率仅在 $P_{2}$ 时低于蓝色折线代 表的简单组命中率, 说明布局预测功能在复杂组 中表现更好. 整个过程中, 平均预测命中率最高为 0.61 , 最低为 0.48 .



a. 简单组命中次数柱状图

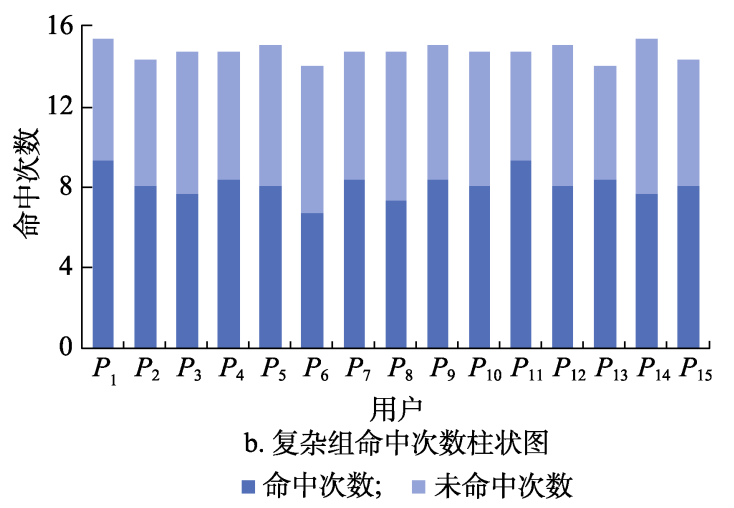

图 12 实验 1 平均命中次数柱状图

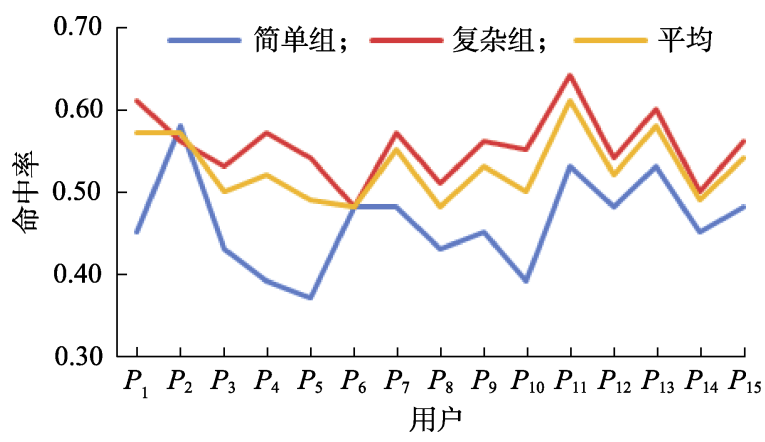

图 13 实验 1 平均命中率折线图

\subsection{3 预测辅助效率分析}

布局预测方法是否能够提高用户的布局效率 的主要衡量指标为时上和操作上的效率. 为此, 本 文设计了对比实验，以比较用户使用或不使用布 局预测方法完成布局需要的时间和操作次数.

对于每次实验，参与者根据数据序列所示的 顺序绘制元素. 对于指定的数据模板, 参与者都需 要绘制 2 次, 一次使用预测功能, 另一次则不使用 预测功能. 如果同一个数据绘制 2 次, 后一次绘制 很容易因为前一次的绘制经验对完成时间和操作 次数产生影响. 为避免这种影响, 系统在每次实验 中从测试集中抽取 10 组数据, 这 10 组数据来自 5 个不同的模板, 其中 2 个来自简单模板, 3 个来自 复杂模板. 这 10 组数据中每 2 组数据来自相同模 板, 但是 2 组数据并不完全相同(数据中的元素位 置或顺序都可能不同). 参与者使用这 2 组数据进 行对比实验, 并且 2 次实验中使用和不使用预测功 能的顺序随机指定.

系统将记录使用预测功能和不使用预测功能 完成这项实验所使用的时间和操作次数. 其中完 成布局的时间表示的是第 1 次布置元素开始到最 后一个元素布置完成的这段时间. 操作次数表示 在完成布局的这段时间中，用户拖动元素、点击元 素等有效的鼠标事件. 不使用和使用预测功能完 成布局的时间差值表示其能为用户节省的时间， 通过计算节省的时间在不使用预测功能完成布局 的时间中的比重, 可以较为直观地看出预测功能 提升的效率.

实验结果统计如图 14 所示, 针对简单和复杂 模板分别进行统计. 在布局完成时间对比实验中, 简单组平均最低使用 $46.12 \mathrm{~s}$, 平均最高使用 $61.51 \mathrm{~s}$; 复杂组最低使用 $67.67 \mathrm{~s}$, 最高使用 $92.05 \mathrm{~s}$. 简单组 最高节省时间为 $13.51 \mathrm{~s}$, 最低为 $7.23 \mathrm{~s}$; 复杂组最 高节省时间为 $28.31 \mathrm{~s}$, 最低为 $15.33 \mathrm{~s}$. 对比图 $14 \mathrm{a}$ 和图 $14 \mathrm{~b}$ 可知, 复杂组节省的时间比例比简单组略 

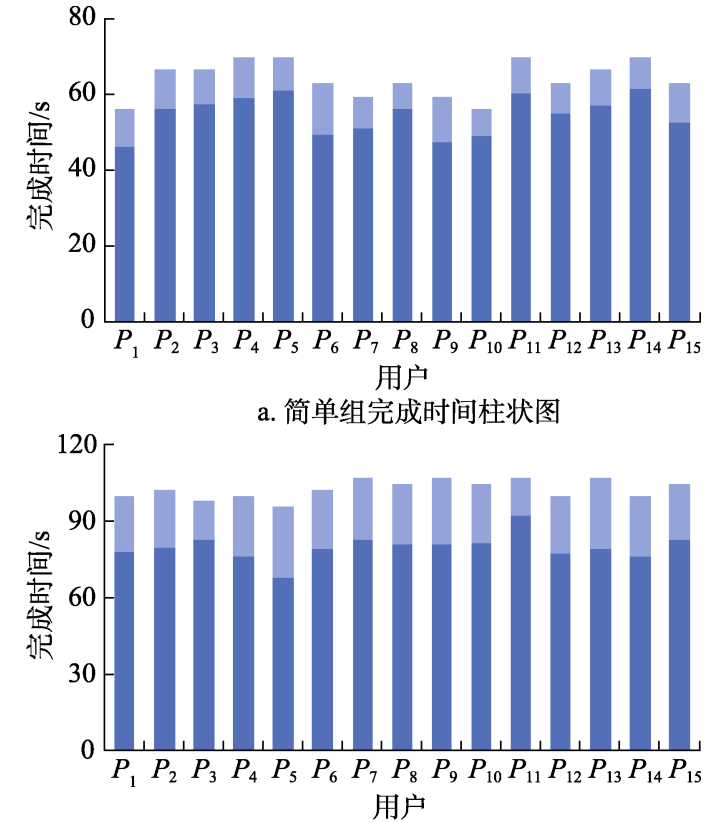

b. 复杂组完成时间柱状图

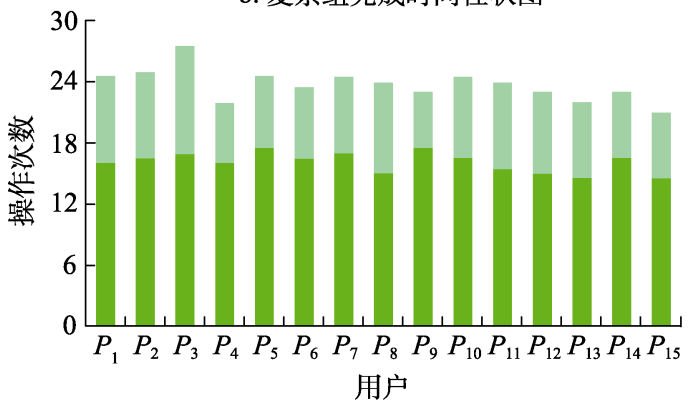

c. 简单组操作次数柱状图

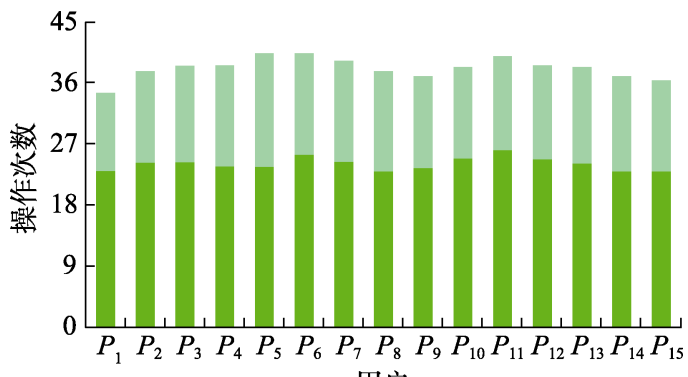

用户

d. 复杂组操作次数柱状图

— 使用预测完成时间; - 节省时间

- 使用预测操作次数; 节省操作次数

图 14 实验 2 完成时间与操作次数柱状图

高. 在操作次数对比实验中, 使用布局预测功能 时, 简单组最高操作次数为 17.5 , 最低为 14.5 ; 复 杂组最高为 26 , 最低为 23 . 根据 $14 \mathrm{c}$ 和图 $14 \mathrm{~d}$ 可知, 简单组与复杂组节省的操作数均较高. 其中复杂 组节省的操作数比例略高于简单组. 各个用户的 提升时间效率比例与操作效率比例如图 15 所示, 无论是操作次数指标还是完成时间指标, 从复杂 组和简单组提升效率比例来看, 复杂组提升效率 更为明显. 在整个实验中, 平均为用户节省的时间
比例最高为 0.24 , 最低为 0.12 . 平均为用户节省的 操作次数比例最高为 0.37 , 最低为 0.32 .


用户

a. 减少的时间比例折线图

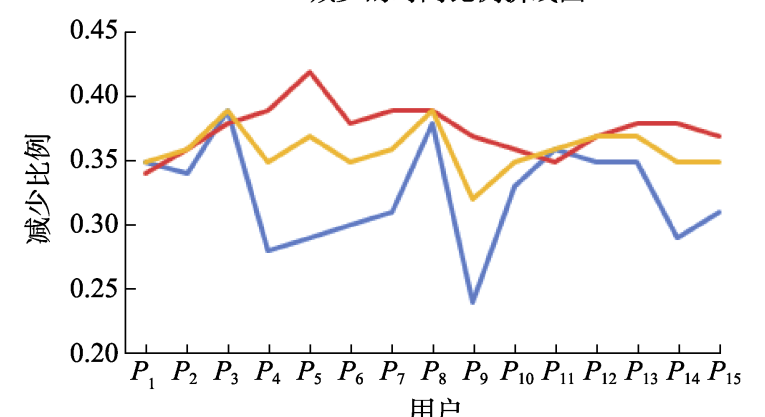

b. 减少的操作数目比例折线图

简单组; 一复杂组; 一 平均

图 15 实验 2 完成时间与操作次数减少比例折线图

此外, 本文对完成时间和操作次数指标进行 方差分析. 表 1 表 8 列出了详细的分析数据. 表格 中的数据均只保留 2 位小数. 表 1 和表 3 简单组中 使用预测完成布局的平均时间为 $54.57 \mathrm{~s}$, 方差为 25.32 , 不使用预测完成时间为 $64.17 \mathrm{~s}$, 方差为 23.92 , 平均节省时间 $9.60 \mathrm{~s}$; 复杂组中使用预测完 成时间为 $79.78 \mathrm{~s}$, 方差为 26.21 , 不使用预测完成 时间为 $102.98 \mathrm{~s}$, 方差为 13.90 , 平均节省时间 $23.20 \mathrm{~s}$. 按照使用和不使用预测功能分类, 简单组 和复杂组的检查统计量(见表 2 和表 4)较大, 表示 简单组与复杂组组间变异大于组内变异, 各组间 的差异远超出总期望值离差, 代表各组的平均数 存在明显差异. 并且 $p$ 值即观测到的显著水平均近 似为 0 , 因此, 检查统计量达到了统计上的意义. 此外, 复杂组中使用预测完成布局的时间的方差 比简单组小, 总体表现更为稳定. 复杂组平均节约 的时间远大于简单组.

表 1 使用预测完成时间统计

\begin{tabular}{rcrcc}
\hline 分组 & 观测数 & 总时间 $/ \mathrm{s}$ & 平均时间 $/ \mathrm{s}$ & 方差 \\
\hline 简单 & 15 & 818.50 & 54.57 & 25.32 \\
复杂 & 15 & 1196.71 & 79.78 & 26.21 \\
\hline
\end{tabular}


表 2 使用预测完成时间 ANOVA

\begin{tabular}{lrrrrrr}
\hline 差异源 & 平方和 & 自由度 & 均方 & 检查统计量 & $p$ 值 & 临界值 \\
\hline 组间 & 4768.09 & 1 & 4768.09 & 185.07 & $7.31 \times 10^{-14}$ & 4.20 \\
组内 & 721.40 & 28 & 25.76 & & & \\
\hline 总计 & 5489.49 & 29 & & & & \\
\hline
\end{tabular}

表 3 不使用预测完成时间统计

\begin{tabular}{ccccc}
\hline 分组 & 观测数 & 总时间 $/ \mathrm{s}$ & 平均时间 $/ \mathrm{s}$ & 方差 \\
\hline 简单 & 15 & 962.50 & 64.17 & 23.92 \\
复杂 & 15 & 1544.73 & 102.98 & 13.90 \\
\hline
\end{tabular}

表 4 不使用预测完成时间 ANOVA

\begin{tabular}{lrrrrrr}
\hline 差异源 & 平方和 & 自由度 & 均方 & 检查统计量 & $p$ 值 & 临界值 \\
\hline 组间 & 11299.73 & 1 & 11299.73 & 597.62 & $1.98 \times 10^{-20}$ & 4.20 \\
组内 & 721.40 & 28 & 25.76 & & & \\
\hline 总计 & 5489.49 & 29 & & & & \\
\hline
\end{tabular}

表 5 使用预测操作次数统计

\begin{tabular}{ccccc}
\hline 分组 & 观测数 & 总次数 & 平均次数 & 方差 \\
\hline 简单 & 15 & 241.50 & 16.10 & 1.00 \\
复杂 & 15 & 360.38 & 24.03 & 0.83 \\
\hline
\end{tabular}

表 6 使用预测操作次数 ANOVA

\begin{tabular}{lrrrrrr}
\hline 差异源 平方和 & 自由度 & 均方 & 检查统计量 & $p$ 值 & 临界值 \\
\hline 组间 & 471.08 & 1 & 471.08 & 511.57 & $1.57 \times 10^{-19}$ & 4.20 \\
组内 & 25.78 & 28 & 0.92 & & & \\
\hline 总计 & 496.87 & 29 & & & & \\
\hline
\end{tabular}

表 7 不使用预测操作次数统计

\begin{tabular}{ccccc}
\hline 分组 & 观测数 & 总次数 & 平均次数 & 方差 \\
\hline 简单 & 15 & 356.00 & 23.73 & 2.39 \\
复杂 & 15 & 573.06 & 38.20 & 2.41 \\
\hline
\end{tabular}

表 8 不使用预测操作次数 ANOVA

\begin{tabular}{crrrrrr}
\hline 差异源 & 平方和 & 自由度 & 均方 & 检查统计量 & $p$ 值 & 临界值 \\
\hline 组间 & 1570.50 & 1 & 1570.50 & 654.41 & $5.84 \times 10^{-21}$ & 4.20 \\
组内 & 67.20 & 28 & 2.40 & & & \\
\hline 总计 & 1637.70 & 29 & & & & \\
\hline
\end{tabular}

操作次数指标提升的效率更高的原因是简单 组的预测命中率相对于复杂组较低, 用户在实际 操作中使用预测功能一次所需要的操作为 1 , 而对 于未命中的元素, 使用其他辅助对齐工具需要进 行的操作有创建和对齐(命令对齐或捕捉对齐) 2 个 动作, 因此操作次数至少会增加 1. 实际操作时对
齐往往需要进行多次尝试, 因此, 操作次数会更多 一些. 并且, 由于系统简化了用户的操作, 同时系 统界面提供的网格线一定程度上限制了用户创建 元素的范围, 用户在某个网格中创建元素并将元 素与其他元素对齐的操作变得更为简单. 因此实 际情景下不使用预测功能时的操作次数将大于实 验中的数据.

\subsection{4 预测鲁棒性分析}

用户在设计布局时总会面临不一样的设计内 容. 为进一步了解布局预测方法在面对新数据时 所发挥的辅助效果, 本文设计了实验以测试布局 预测方法在真实使用场景中的表现. 如果让用户 自由地完成布局设计，不仅各个用户的布局很难 形成对比, 实验也很难评估出布局预测方法在布 局设计过程中发挥的作用. 因此, 采取指定布局主 题的方式, 即指定一个流程图模板, 用户需要绘制 出与此模板不同的变体. 这项实验将评估布局预 测方法的鲁棒性. 本实验所针对的布局类型并未 出现在训练数据中.

从原始模板中抽取了 10 组未出现在训练集中 的模板, 其中包含了 5 组较为简单的模板和 5 组较 为复杂的模板. 在每次实验中参与者都会收到 2 组 简单模板和 3 组复杂模板, 这些模板随机分发. 在 实验中, 系统并不会为参与者提供这些模板的绘 制序列. 参与者需要在设计过程中指定绘制元素的 顺序. 系统将记录参与者使用布局预测结果的次数 和元素总个数. 最后计算出使用预测结果占总元素 个数的比例, 以此评估方法在实验中发挥的作用.

实验结果统计如图 16 所示. 可以看出, 简单 组预测命中次数最高为 3.5 次, 最低为 2 次; 复杂 组最高为 6.34 次, 最低为 5 次. 简单组和复杂组的 命中次数比例比前面的预测命中率实验有所下降. 如图 17 所示, 在整个实验过程中平均命中率最高 为 0.50 , 最低为 0.42 . 导致命中率下降的原因可能 有: 训练网络的数据是自动生成的, 并不一定适合 用户的实际布局习惯; 用户可能会构造一些更为 奇怪的布局导致网络无法成功预测.

从 3 个用户调查实验结果看出, 复杂组在各项 实验指标上均比简单组表现更好. 其原因是: 简单 组的序列长度比复杂组的短, 网络从中获取到的 序列信息更少, 制约新元素出现的位置的元素较 少, 所以预测结果面向的求解范围更广, 从而较难 给出准确的预测结果; 而复杂组序列长度较长, 新 元素的制约元素更多, 产生的约束更强, 面向的求 解范围更小, 更容易得出准确的预测结果. 


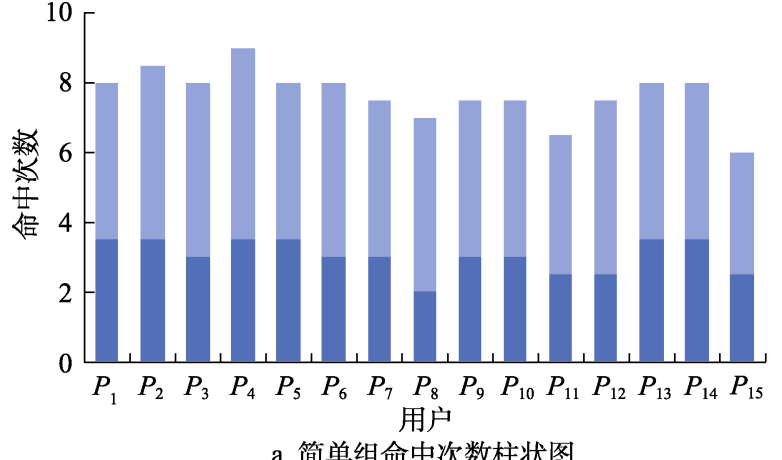

a. 简单组命中次数柱状图

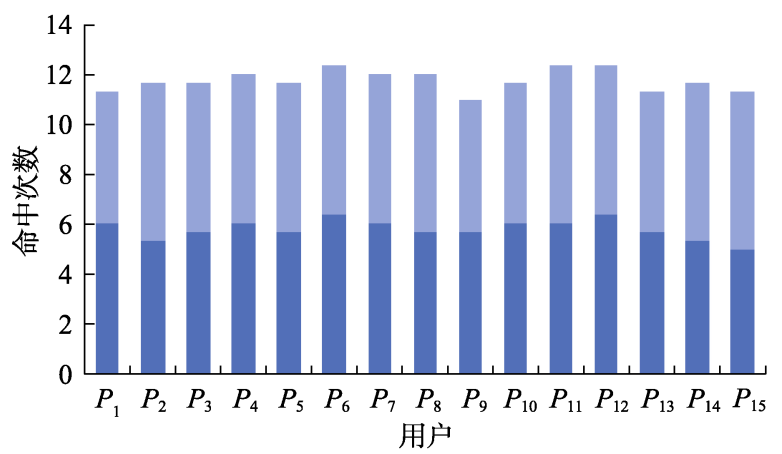

b. 复杂组命中次数柱状图

- 命中次数; 未命中次数

图 16 实验 3 平均命中次数柱状图

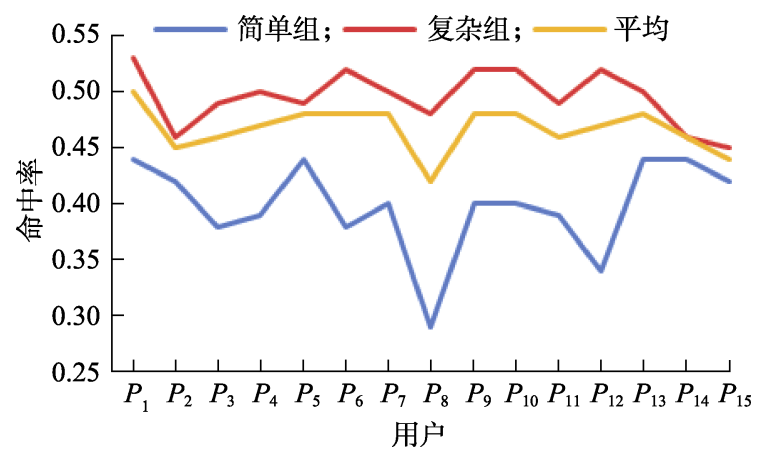

图 17 实验 3 用户预测命中率折线图

\section{5 结 语}

本文提出了一个基于预测的布局方法，以帮 助用户在布局时减少重复和无用的操作. 将布局 预测方法输出的多个结果通过可视化的方式展示 出来, 用户可直接对比不同方案间的差别. 实验结 果表明, 本文方法能够有效预测布局, 并且在设计 效率上有一定的提升. 本文主要贡献如下.

(1) 提出的基于预测的布局方法在传统的布 局流程上额外增加预测功能, 对新元素的位置进 行预测，提升了用户的布局效率.

（2）尝试使用神经网络辅助用户布局，其网格 化数据策略能将多对一的数据扩展为多对多的形
式. 网络模型预测出多个结果为用户提供多个候 选布局，能帮助用户更快地完成布局设计.

(3) 创建了一个用于训练神经网络的数据集. 从专业网站上获取到数据模板, 并对模板进行数 据增强以获取更多的训练数据.

(4) 开发了一个布局预测系统, 可将预测结果 直接可视化出来, 不仅能为用户提供多个候选布 局的样例, 而且预测结果之间还能直观地进行视 觉对比，方便用户从中选择出理想的布局.

本文提出的布局预测方法也存在一些限制. 例如, 网格若发生改变, 则需要重新训练网络模 型. 如果能获取到真实的用户数据, 本文方法可扩 展到预测用户个性化的布局行为.

\section{参考文献(References):}

[1] Mackay W E, Ratzer A V, Janecek P. Video artifacts for design: bridging the Gap between abstraction and detail[C] //Proceedings of the 3rd Conference on Designing Interactive Systems: Processes, Practices, Methods, and Techniques. New York: ACM Press, 2000: 72-82

[2] Felice M C, Maudet N, Mackay W E, et al. Beyond snapping: persistent, tweakable alignment and distribution with StickyLines[C] //Proceedings of the 29th Annual Symposium on User Interface Software and Technology. New York: ACM Press, 2016: 133-144

[3] Xu P F, Fu H B, Tai C L, et al. GACA: group-aware command-based arrangement of graphic elements[C] //Proceedings of the 33rd Annual ACM Conference on Human Factors in Computing Systems. New York: ACM Press, 2015: 2787-2795

[4] Sutherland I E. Sketchpad a man-machine graphical communication system[J]. Simulation, 1964, 2(5): R-3-R-20

[5] Bier E A, Stone M C. Snap-dragging[C] //Proceedings of the 13th Annual Conference on Computer Graphics and Interactive Techniques. New York: ACM Press, 1986: 233-240

[6] Fernquist J, Shoemaker G, Booth K S. “Oh snap”-helping users align digital objects on touch interfaces[C] //Proceedings of the 13th IFIP TC 13 International Conference on Human-Computer Interaction. Heidelberg: Springer, 2011: 338-355

[7] Baudisch P, Cutrell E, Hinckley K, et al. Snap-and-go: helping users align objects without the modality of traditional snapping[C] //Proceedings of the SIGCHI Conference on Human Factors in Computing Systems. New York: ACM Press, 2005: 301-310

[8] Nelson G. Juno, a constraint-based graphics system[C] //Proceedings of the 12th Annual Conference on Computer Graphics and Interactive Techniques. New York: ACM Press, 1985: 235-243

[9] van Wyk C J. A high-level language for specifying pictures[J]. ACM Transactions on Graphics, 1982, 1(2): 163-182

[10] Dwyer T, Marriott K, Wybrow M. Dunnart: a constraint-based network diagram authoring tool[C] //Proceedings of the 16th 
International Symposium on Graph Drawing. Heidelberg: Springer, 2008: 420-431

[11] Kurlander D, Feiner S. Inferring constraints from multiple snapshots[J]. ACM Transactions on Graphics, 1993, 12(4): 277-304

[12] Igarashi T, Matsuoka S, Kawachiya S, et al. Interactive beautification: a technique for rapid geometric design[C] //Proceedings of the 10th Annual ACM Symposium on User Interface Software and Technology. New York: ACM Press, 1997: 105-114

[13] Chok S S, Marriott K. Automatic construction of intelligent diagram editors[C] //Proceedings of the 11th Annual ACM Symposium on User Interface Software and Technology. New York: ACM Press, 1998: 185-194

[14] O'Donovan P, Agarwala A, Herzmann A. DesignScape: design with interactive layout suggestions[C] //Proceedings of the 33rd Annual ACM Conference on Human Factors in Computing Systems. New York: ACM Press, 2015: 1221-1224

[15] Xu P F, Yan G H, Fu H B, et al. Global beautification of 2D and 3D layouts with interactive ambiguity resolution[J]. IEEE Transactions on Visualization and Computer Graphics, 2021, 27(4): 2355-2368

[16] Wybrow M, Marriott K, Mciver L, et al. Comparing usability of one-way and multi-way constraints for diagram editing[J]. ACM Transactions on Computer-Human Interaction, 2008, 14(4): Article No.19

[17] Gleicher M. Briar: a constraint-based drawing program[C] // Proceedings of the SIGCHI Conference on Human Factors in Computing Systems. New York: ACM Press, 1992: 661-662

[18] Ryall K, Marks J, Shieber S. An interactive constraint-based system for drawing graphs[C]//Proceedings of the 10th Annual ACM Symposium on User Interface Software and Technology. New York: ACM Press, 1997: 97-104
[19] Masui T. HyperSnapping[C] //Proceedings of the IEEE Symposia on Human Centric Computing Languages and Environments. Los Alamitos: IEEE Computer Society Press, 2001: 188-194

[20] Li J N, Yang J M, Hertzmann A, et al. LayoutGAN: generating graphic layouts with wireframe discriminators[OL]. [2020-12-01]. https://arxiv.org/abs/1901.06767

[21] Lee H Y, Jiang L, Essa I, et al. Neural design network: graphic layout generation with constraints[OL]. [2020-12-01]. https://arxiv.org/abs/1912.09421

[22] Zeidler C, Weber G, Stuerzlinger W, et al. Automatic generation of user interface layouts for alternative screen orientations[C] //Proceedings of IFIP Conference on Human-Computer Interaction. Heidelberg: Springer Press, 2017: 13-35

[23] Jiang Y, Du R F, Lutteroth C, et al. ORC layout: adaptive GUI layout with OR-constraints[C] //Proceedings of the CHI Conference on Human Factors in Computing Systems. New York: ACM Press, 2019: Article No.413

[24] Xu K, Ba J L, Kiros R, et al. Show, attend and tell: neural image caption generation with visual attention[C]//Proceedings of the 32nd International Conference on International Conference on Machine Learning. New York: ACM Press, 2015: 20482057

[25] Simonyan K, Zisserman A. Very deep convolutional networks for large-scale image recognition[OL]. [2020-12-01]. https:// rxiv.org/abs/1409.1556

[26] He K M, Zhang X Y, Ren S Q, et al. Deep residual learning for image recognition[C] //Proceedings of the IEEE Conference on Computer Vision and Pattern Recognition. Los Alamitos: IEEE Computer Society Press, 2016: 770-778

[27] Glaser B, Strauss A. The discovery of grounded theory: strategies for qualitative research[M]. Chicago: Aldine Transaction, 2009 